\title{
Some Fixed Point Theorems for pair mapping in Complex Valued b-Metric Space
}

\author{
S. K. Tiwari ${ }^{1}$, Y.K.Yadav ${ }^{2}$ \\ ${ }^{I}$ Department of Mathematics, DR. C.V. Raman University, Bilaspur, Chhattisgarh-495113, India \\ ${ }^{2}$ Department of Mathematics (M. Phil Scholar) DR. C.V. Raman University, Bilaspur, Chhattisgarh-495113, \\ India
}

\begin{abstract}
In this paper, we prove some common fixed point results for pair of rational type of contractive mappings in the setting of complex valued b-metric spaces. Our results extend, generalize and improve the corresponding results of $A$. K.Dubey' [48].
\end{abstract}

Key word: Complex valued b-metric space; common fixed point; Rational expression type of contractive mappings.

\section{Introduction.}

Fixed point theorem general known as the Banach contraction mapping theorem. Banach Contraction principle [4] is a basic result in fixed point theory. Later, a larger number of articles have been devoted to the improvement and generalization of the Banach Contraction Principle by using different form of contraction condition in various spacesIn this theory contraction is one of main tools to prove the existence and uniqueness of a fixed point. Banach Contraction Principle which give an answer on existence and uniqueness of a solution of an operator equation

$$
T x=x .
$$

This equation is the most widely used for fixed point theorem in all analysis. This principle is contractive in nature and is one of the most useful tools in the study a non-linear equation. There are a lot of generalizations of the Banach Contraction Mapping Principle in the iterative.

The Banach Contractive Principle was used to establish the existence of a unique solution for a nonlinear integral equation [4]. There are many generalizations of the Banach Contraction Principle particularly in the metric space see for instance([ 1][2][3],[7][12][16],[19][20][21],[22][23][24]). There are a lot of generalization of this principle has been obtained in several directions.

In 1989, Bakhtin [25] introduce the concept of b-metric space. In 1993, Czerwik [26] online the results of bmetric space which is a generalized the famous Banach contractive Principle in metric space. Using this idea researcher presented generalization of the renowned Banach Fixed Point Theorem in the b-metric space for ([27],[28][29]). Many authers have studied the extension of Fixed Point Theorem in b-metric space, see for instance ([30],[31,32,33],[34],[35],[36],[37][38],[39],,[40,41][42][43],[44],[45]\&[46]\&[47]).

In 1978, Feisher and khan [9] generalized the Banach Contraction Principle with rational expression and proved some fixed and common fixed point theorems.

Recently Azam et al. [2] introduced the notation of complex valued metric space and proved some common fixed point theorems for mapping satisfying rational inequality which are not meaningful in cone metric space.

In the same way, various authers have studied and prove the fixed point result for mapping satisfying different type contractive conditions in the framework of complex valued metric space (see [15] ,[8], [5] ,[18],[17] ). In 2013 Rao et al [13] introduce the concept of complex valued b- metric space which was more general then the well known complex valued metric space. In sequal A.A. Mukheimer [11] obtained common fixed point result satisfying certain rational expression in complex valued b-metric space. In sequel A.K. Dubey, etal. (2015) obtain fixed point results for the mapping satisfied rational expression in complex valued b-metric space. The main purpose of this paper is the present common fixed point results of two self mapping satisfying rational expression in complex valued b-metric spaces our results in this paper are generalization of work done. A.K. Dubey, et al in [48].

\section{Preliminaries}

Definition2.1 (see [30]) Let $X$ be a non empty set and let $s \geq 1$ be a given real number .A function $d: X \times X \rightarrow$ $[0, \infty)$ is called $\mathrm{b}$-metric if for all $x, y, z \in X$. The following condition are satisfied.

(i) $d(x, y)=0$ iff $x=y$

(ii) $d(x, y)=d(y, x)$ 
(iii) $d(x, y) \leq S[d(x, z)+d(z, y)]$.

The pair $(x, d)$ is called a b-metric space. The numbers $\geq 1$ is called the coefficient of $(X, d)$.

Example 2.1.1(see [10]) Let $X=\{-1,0,1\}$.Define mapping $d: X \times X \rightarrow R^{+}$by

$$
d(x, y)=d(y, x) \forall x, y \in X .
$$

I $\quad d(x, x)=0, d(-1,0)=4, d(-1,1)=1, d(0,1)=2$

Then $d: X \times X \rightarrow R$ or $(X, d)$ is b-metric space, but not metric space, since the triangle inequality is not satisfied indeed.

$$
d(-1,0)+d(0,1)=4+2=6>1=d(-1,1)
$$

and

are both true but

$$
d(-1,1)+d(-1,0)=1+4=5>2=d(0,1)
$$

$$
d(-1,1)+d(0,1)=1+2=3<4=d(-1,1)
$$

is not true. So $(X, d)$ is b-metric space with $\mathrm{S}=4 / 3$.

Example 2.1.2 (see [10]) Let $X=[0,1]$ and $d: X \times X \rightarrow[0, \infty]$ be defined by $d(x, y)=(x-y)^{2} \forall x, y \in X$. Clearly $(X, d)$ is b-metric space.

Example2.1.3 (see [11]) Let $(X, d)$ be a metric space and $d^{*}(x, y)=(d(x, y))^{p}$ with $p \geq 1$ is a real number. Then $\left(X, d^{*}\right)$ is a b-metric space with $S=2^{p-1}$.

An ordinary metric $d$ is a real valued function from a set $X \times X$ into $R$ where $X$ is non empty set that is $d: X \times X \rightarrow R$. A complex number $z \in \mathbb{C}$ is an ordered pair of real numbers where first co-ordinate is called $\operatorname{Re}(z)$ and second co-ordinate is called $\operatorname{Im}(z)$. Thus a complex valued metric space $d$ is a function from a set $X \times X$ into $\mathbb{C}$, where $X$ is the non-empty set and $\mathbb{C}$ is the set of complex number .

That is $d: X \times X \rightarrow \mathbb{C}$. Let $z_{1}, z_{2} \in \mathbb{C}$ difine a partial order $\leq$ on $\mathbb{C}$ as follows

$z_{1} \leq z_{2}$ iff $\operatorname{Re}\left(z_{1}\right) \leq \operatorname{Re}\left(z_{2}\right), \operatorname{Im}\left(z_{1)} \leq \operatorname{Im}\left(z_{2}\right)\right.$.

It follow that $z_{1} \leq z_{2}$ if one of the following condition are satisfied:

(i) $\operatorname{Re}\left(z_{1}\right)=\operatorname{Re}\left(z_{2}\right)$ and $\operatorname{Im}\left(z_{1}\right)<\operatorname{Im}\left(z_{2}\right)$

(ii) $\operatorname{Re}\left(z_{1}\right)<\operatorname{Re}\left(z_{2}\right)$ and $\operatorname{Im}\left(z_{1}\right)=\operatorname{Im}\left(z_{2}\right)$

(iii) $\operatorname{Re}\left(z_{1}\right)<\operatorname{Re}\left(z_{2}\right) \quad$ and $\operatorname{Im}\left(z_{1}\right)<\operatorname{Im}\left(z_{2}\right)$

(iv) $\operatorname{Re}\left(z_{1}\right)=\operatorname{Re}\left(z_{2}\right)$ and $\operatorname{Im}\left(z_{1}\right)=\operatorname{Im}\left(z_{2}\right)$

In (i),(ii),(iii) we have $\left|z_{1}\right| \leq\left|z_{2}\right|$. In (iv), we have $\left|z_{1}\right|=\left|z_{2}\right|$. So $\left|z_{1}\right| \leq\left|z_{2}\right|$ In particular $\left|z_{1}\right| \lesseqgtr\left|z_{2}\right|$ if $z_{1} \neq z_{2}$ and one of (i), (ii), (iii) is satisfy. In this case $\left|z_{1}\right|<\left|z_{2}\right|$. We will write $z_{1}<z_{2}$ iff (iii) satisfy. Further

$$
\begin{aligned}
0 \leq z_{1} z_{2} \Rightarrow\left|z_{1}\right| \leq\left|z_{2}\right| . \\
z_{1} \leq z_{2} \text { and } z_{2}<z_{3} \Rightarrow z_{1}<z_{3} .
\end{aligned}
$$

Definition 2.2 (see [2]) Let $\mathrm{x}$ be a nonempty set. Suppose that the mapping $d: X \times X \rightarrow \mathbb{C}$ satisfies the following conditions:

(i) $d(x, y) \geq 0$ and $d(x, y)=0 \Leftrightarrow x=y$.

(ii) $d(x, y)=d(y, x)$

(iii) $d(x, y) \leq d(x, z)+d(z, y)$

(symmetic)

(the trinagle inequalities).

Then $d$ is called a complex valued metric on $X$ and $(X, d)$ is called a complex valued metric space.

Example 2.2.1(see [8]) Let $X=\mathbb{C}$ Define the mapping $d: X \times X \rightarrow C$ by $d(x, y)=i|x-y|, \forall x, y \in X$. Then $(X, d)$ is complex valued metric space.

Example 2.2.2 (see [19]) Let $X=\mathbb{C}$ Define the mapping $d: X \times X \rightarrow \mathbb{C}$ by

$$
d(x, y)=e^{i k}|x-y| \text { where } k \in R \text { and }, \forall x, y \in X,
$$

Where $k \in\left[0, \frac{\pi}{2}\right], \forall x, y \in X$.Then $(X, d)$ is called a complex valued metric space.

Definition 2.3 (see [4]) Let $X$ be a nonempty set and mapping $d: X \times X \rightarrow \mathbb{C}$ satisfy the following conditions:

(i) $0 \leq d(x, y)$ and $d(x, y)=0$ iff $x=y \quad \forall x, y \in X$.

(ii) $d(x, y)=d(y, x)$

(iii) $d(x, y) \leq S[d((x, z)+d(z, y)]$.

Where $s \geq 1$ is a real number. Then $\mathrm{d}$ is called complex valued metric space and $(X, d)$ is called complex valued $b$-metric space.

Example2.3.1 (see [13]) Let $X=[0,1]$. Define a complex valued metric $d: X \times X \rightarrow \mathbb{C}$ by $d(x, y)=|x-y|^{2}+i|x-y|^{2}, \forall x, y \in X$

Then $(X, d)$ is a complex valued b-metric space with $\mathrm{S}=2$.

Remark 2.4: If $S=1$, then the complex valued $b$-metric space always reduces to a complex valued metric space. Thus every complex valued metric space is a complex valued b-metric space, but not conversely. This generalizes the notation of a complex valued $b$-metric space over complex valued metric space.

Definition 2.5 (see [13]) Let $(X, d)$ be a complex valued b-metric space consider the following: 
(i) A point $x \in X$ is called interior point of a set $A \subset X$ whenever point. There exists $0<r \in C$ such that

$$
B(x, r)=\{y \in X: d(x, y)<r\} \subseteq A .
$$

(ii) A point $x \in X$ is called a limit point of a set $A$ whenever there exists for every $0<r \in C$,

$$
B(x, r) \cap(A-X)=\varnothing \text {. }
$$

(iii) A subset $A \subseteq X$ is called open whenever each element of $A$ is an interior point of $A$.

(iv) A subset $A \subseteq X$ is called closed whenever each element of a $A$ belong to $A$.

(v) A subbasis for a Housdroff topology $\tau$ on $X$ is a family

$$
F=\{B(x, r): x \in X \text { and } 0<r\}
$$

Definition 2.6 (see [13]) Let $(X, d)$ be a complex valued b-metric space and $\left\{x_{n}\right\}$ a sequence in $\mathrm{X}$ and $x \in X$ consider the following:

(i) If for every $c \in C$, with $0<r$, there is $n \in N$ such that for all $d\left(x_{n}, x\right)<c$. Then $\left\{x_{n}\right\}$ is said to be convergent, $\left\{x_{n}\right\}$ coverges to $x$ and $x$ is the limit point of $\left\{x_{n}\right\}$.We donote this by $\lim _{n \rightarrow \infty} x_{n}=x$ or $\left\{x_{n}\right\} \rightarrow$ $x$ as $n \rightarrow \infty$

(ii)If for every $c \in \mathbb{C}$ with $0<r$, there is $n>N d\left(x_{n}, x_{n+m}\right)<r$. where $m \in N$. Then $\left\{x_{n}\right\}$ is said to be Cauchy sequence.

(iii)If every Cuachy sequence in $X$ is convergent, then $(X, d)$ is said to be a complete complex valued b-metric space.

Lemma 2.7(see [13]): let $(X, d)$ be a complex valued b-metric space and Let $\left\{x_{n}\right\}$ be a sequence in $X$. Then $\left\{x_{n}\right\}$ converges to $\mathrm{x}$ if and if only $\mid d\left(x_{n}, x \mid \rightarrow 0\right.$ as $n \rightarrow \infty$.

Lemma 2.8 (see [13]): let $(X, d)$ be a complex valued b-metric space and Let $\left\{x_{n}\right\}$ be a sequence in $X$. Then $\left\{x_{n}\right\}$ is a Cauchy sequence if and if only $\left|d\left(x_{n}, x_{n+m}\right)\right| \rightarrow 0$ as $n \rightarrow \infty$, where $m \in N$.

\section{Main Results}

The following results are generalizations of theorem 7 and 9 of A. K. Dubey [48].

Theorem 3.1 Let $(X, d)$ be a complete complex valued b-metric space with the coefficient $s \geq 1$ and $T_{1}, T_{2}: X \rightarrow X$ be a mapping satisfying the condition:

$$
d\left(T_{1}, x, T_{2} y\right) \leq \frac{\lambda d^{2}(x, y)}{1+d(x, y)}+\mu d\left(y, T_{2}, y\right)
$$

for all $\forall x, y \in X$, where $\lambda, \mu$ are nonnegative reals with $s \lambda+\mu<1$.Then $T_{1}$ and $T_{2}$ have a unique common fixed point in $X$.

Proof. For any arbitrary point, $x_{n} \in X$. Define sequence $\left\{x_{n}\right\}$ in $X$ such that

$$
\begin{aligned}
& x_{2 n+1}=T_{1} x_{2 n} \text { for } n=\{0,1,2,3, \ldots\} \\
& x_{2 n+2}=T_{2} x_{2 n+1} \text { for } n=\{0,1,2,3, \ldots\}
\end{aligned}
$$

Now, we show that the sequence $\left\{x_{n}\right\}$ is Cauchy: Let $x=x_{2 n} \& y=x_{2 n+1}$ in (1) we have

$$
\begin{aligned}
d\left(x_{2 n+1}, x_{2 n+2}\right) & =d\left(T_{1} x_{2 n}, T_{2} x_{2 n+1}\right) \\
& \leq \frac{\lambda d^{2}\left(x_{2 n}, x_{2 n+1}\right)}{1+d\left(x_{2 n}, x_{2 n+1}\right)}+\mu d\left(x_{2 n+1}, T_{2} x_{2 n+1}\right) \\
& =\frac{\lambda d^{2}\left(x_{2 n}, x_{2 n+1}\right)}{1+d\left(x_{2 n}, x_{2 n+1}\right)}+\mu d\left(x_{2 n+1}, x_{2 n+2}\right)
\end{aligned}
$$

Which implies that $\left|d\left(x_{2 n+1}, x_{2 n+2}\right)\right| \leq \lambda \frac{\left|d\left(x_{2 n}, x_{2 n+1}\right)\right|}{\left|1+d\left(x_{2 n}, x_{2 n+1}\right)\right|}\left|d\left(x_{2 n}, x_{2 n+1}\right)\right|+\mu\left|d\left(x_{2 n+1}, x_{2 n+2}\right)\right|$

Since $\left|1+d\left(x_{2 n}, x_{2 n+1}\right)\right|>\left|d\left(x_{2 n}, x_{2 n+1}\right)\right|$, we get

$\left|d\left(x_{2 n+1}, x_{2 n+2}\right)\right| \leq \lambda\left|d\left(x_{2 n}, x_{2 n+1}\right)\right|+\mu\left|d\left(x_{2 n+1}, x_{2 n+2}\right)\right|$

and hence

$$
\left|d\left(x_{2 n+1}, x_{2 n+2}\right)\right| \leq \frac{\lambda}{1-\mu}\left|d\left(x_{2 n}, x_{2 n+1}\right)\right|
$$

Similarly, we obtain

$$
\left|d\left(x_{2 n+2}, x_{2 n+3}\right)\right| \leq \frac{\lambda}{1-\mu}\left|d\left(x_{2 n+1}, x_{2 n+2}\right)\right| .
$$

Since $s \lambda+\mu<1$ and $s \geq 1$, we get $\lambda+\mu<1$.

Therefore, with $\delta=\frac{\lambda}{1-\mu}<1$ and for all $n \geq 0$. and consequently, we have

$$
\begin{gathered}
\left|d\left(x_{2 n+1}, x_{2 n+2}\right)\right| \leq \delta\left|d\left(x_{2 n}, x_{2 n+1}\right)\right| \leq \delta^{2}\left|d\left(x_{2 n-1}, x_{2 n}\right)\right| \leq \cdots \leq \delta^{2 n+1}\left|d\left(x_{0}, x_{1}\right)\right| \\
\left|d\left(x_{2 n+1}, x_{2 n+2}\right)\right| \leq \delta\left|d\left(x_{2 n}, x_{2 n+1}\right)\right| \leq \delta^{2}\left|d\left(x_{2 n-1}, x_{2 n}\right)\right| \leq \cdots \leq \delta^{2 n+1}\left|d\left(x_{0}, x_{1}\right)\right|
\end{gathered}
$$

Thus for any $m>n, m, n \in \mathbb{N}$ and since $s \delta=\frac{s \lambda}{1-\mu}<1$, we get

$$
\begin{aligned}
\left|d\left(x_{2 n}, x_{2 m}\right)\right| & \leq s\left|d\left(x_{2 n}, x_{2 n+1}\right)\right|+s\left|d\left(x_{2 n+1}, x_{2 m}\right)\right| \\
& \leq s\left|d\left(x_{2 n}, x_{2 n+1}\right)\right|+s^{2}\left|d\left(x_{2 n+1}, x_{2 n+2}\right)\right|+s^{2}\left|d\left(x_{2 n+2}, x_{2 m}\right)\right| \\
& \leq s\left|d\left(x_{2 n}, x_{2 n+1}\right)\right|+s^{2}\left|d\left(x_{2 n+1}, x_{2 n+2}\right)\right|+s^{3}\left|d\left(x_{2 n+2}, x_{2 n+3}\right)\right| \\
& +s^{3}\left|d\left(x_{2 n+3}, x_{2 m}\right)\right|
\end{aligned}
$$


$\left|d\left(x_{2 n}, x_{2 m}\right) \dot{\mid} \leq s\right| d\left(x_{2 n}, x_{2 n+1}\right)\left|+s^{2}\right| d\left(x_{2 n+1}, x_{2 n+2}\right)\left|+s^{3}\right| d\left(x_{2 n+2}, x_{2 n+3}\right) \mid+\cdots$
$\ldots .+s^{2 m-2 n-1}\left|d\left(x_{2 m-2}, x_{2 m-1}\right)\right|+s^{2 m-2 n}\left|d\left(x_{2 m-1}, x_{2 m}\right)\right|$

By using (9), we get

$$
\ldots .+s^{2 m-2 n-1}\left|d\left(x_{2 m-2}, x_{2 m-1}\right)\right|+s^{2 m-2 n}\left|d\left(x_{2 m-1}, x_{2 m}\right)\right|
$$

$\left|d\left(x_{2 n}, x_{2 m}\right)\right| \leq s \delta^{2 n}\left|d\left(x_{0}, x_{1}\right)\right|+s^{2} \delta^{2 n+1}\left|d\left(x_{0}, x_{1}\right)\right|+s^{3} \delta^{2 n+2}\left|d\left(x_{0}, x_{1}\right)\right|+\cdots$

Therefore,

$$
=\sum_{i=1}^{2 m-2 n} s^{i} \delta^{i+2 n-1}\left|d\left(x_{0}, x_{1}\right)\right| \text {. }
$$

$$
\begin{aligned}
\left|d\left(x_{2 n}, x_{2 m}\right)\right| & =\sum_{i=1}^{2 m-2 n} s^{i+2 n-1} \delta^{i+2 n-1}\left|d\left(x_{0}, x_{1}\right)\right| \\
& =\sum_{t=n}^{2 m-1} s^{t} \delta^{t}\left|d\left(x_{0}, x_{1}\right)\right| \\
& \leq \sum_{t=2 n}^{\infty}(s \delta)^{t}\left|d\left(x_{0}, x_{1}\right)\right| \\
& =\frac{(s \delta)^{2 n}}{1-(s \delta)}\left|d\left(x_{0}, x_{1}\right)\right|
\end{aligned}
$$

and hence

$$
\left|d\left(x_{2 n}, x_{2 m}\right)\right| \leq \frac{(s \delta)^{2 n}}{1-(s \delta)}\left|d\left(x_{0}, x_{1}\right)\right| \rightarrow 0 \text { as } n, m \rightarrow \infty
$$

Thus, $\left\{x_{n}\right\}$ is a Cauchy sequence in $X$. Since $X$ is complete, there exists some $u \in X$ such that $x_{n} \rightarrow u$ as $n \rightarrow \infty$. Suppose that is not possible; then there exists $z \in X$ such that

Now,

$$
\left|d\left(u, T_{1} u\right)\right|=|z|>0
$$

$$
\begin{aligned}
z & =d(u, T u) \leq s d\left(u, x_{2 n+2}\right)+s d\left(x_{2 n+2}, T_{1} u\right) \\
& =s d\left(u, x_{2 n+2}\right)+s d\left(T_{2} x_{2 n+2}, T_{1} u\right) \\
& \leq s d\left(u, x_{2 n+2}\right)+\frac{s \lambda d^{2}\left(x_{2 n+1}, u\right)}{1+d\left(x_{2 n+1}, u\right)}+s \mu d\left(u, T_{1} u\right)
\end{aligned}
$$

which implies that

$$
|z|=\left|d\left(u, T_{1} u\right)\right| \leq s\left|d\left(u, x_{2 n+2}\right)\right|+\frac{s \lambda\left|d^{2}\left(x_{2 n+1}, u\right)\right|}{1+\left|d\left(x_{2 n+1}, u\right)\right|}+s \mu\left|d\left(u, T_{1} u\right)\right| .
$$

Taking the limit of (16) as $n \rightarrow \infty$, we obtain that $|z|=\left|d\left(u, T_{1} u\right)\right| \leq 0$, a contradiction with (14).

So $|z|=0$. Hence $T_{1} u=u$.

Similarly, we can show that $T_{1} u=u$.

Now show that $T_{1}$ and $T_{2}$ have unique common fixed point of $T_{1}$ and $T_{2}$. To show this, assume that $u^{*}$ is another fixed point of $T_{1}$ and $T_{2}$.Then,

$$
d\left(u, u^{*}\right)=d\left(T_{1} u, T_{2} u^{*}\right) \leq \frac{\lambda d^{2}\left(u, u^{*}\right)}{1+d\left(u, u^{*}\right)}+\mu d\left(u^{*}, T_{2} u^{*}\right)
$$

So

$$
\left|d\left(u, u^{*}\right)\right| \leq \lambda \frac{\left|d^{2}\left(u, u^{*}\right)\right|}{\left|1+d\left(u, u^{*}\right)\right|}\left|d\left(u, u^{*}\right)\right|+\mu\left|d\left(u^{*}, T_{2} u^{*}\right)\right|
$$

Since

$$
\left|1+d\left(u, u^{*}\right)\right|>\left|d\left(u, u^{*}\right)\right|
$$

Therefore

$$
\begin{array}{r}
\left|d\left(u, u^{*}\right)\right|<\lambda\left|d\left(u, u^{*}\right)\right|+\mu\left|d\left(u^{*}, u^{*}\right)\right| \\
=\lambda\left|d\left(u, u^{*}\right)\right|, \text { a contradiction. }
\end{array}
$$

So, $u=u^{*}$, which proves the uniqueness of fixed point in $X$. This completes the proof.

Theorem 3.2 Let $(X, d)$ be a complete complex valued b-metric space with the coefficient $s \geq 1$ and $T_{1}, T_{2}: X \rightarrow X$ be a mapping satisfying

$$
d\left(T_{1} x, T_{2} y\right) \leq \lambda d(x, y)+\frac{\mu d\left(x, T_{1} x\right) d\left(y, T_{2} y\right)}{d\left(x, T_{2} y\right)+d\left(y, T_{1} x\right)+d(x, y)^{\prime}}
$$

for all $x, y \in X$ such that $x \neq y, d\left(x, T_{2} y\right)+d\left(y, T_{1} x\right)+d(x, y) \neq 0$, where $\lambda, \mu$ are nonnegative reals with $s \lambda+\mu<1$ or $d\left(T_{1} x, T_{2} y\right)=0$ if $d\left(x, T_{2} y\right)+d\left(y, T_{1} x\right)+d(x, y)=0$. Then $T_{1} \& T_{2}$ have a unique common fixed point in $X$.

Proof. For any arbitrary point, $x_{0} \in X$.Define sequence $\left\{x_{n}\right\}$ in $X$ such that

$\begin{array}{cc}x_{2 n+1}=T_{1} x_{2 n} \quad \text { for } \\ x_{2 n+2}=T_{2} x_{2 n+1} & \text { for } n=(0,1,2,3 \ldots)\end{array}$

Now, we show that the sequence $\left\{x_{n}\right\}$ is Cauchy: Let $x=x_{2 n} \& y=x_{2 n+1}$ in (21) we have

$$
\begin{aligned}
d\left(x_{2 n+1}, x_{2 n+2}\right) & =d\left(T_{1} x_{2 n}, T_{2} x_{2 n+1}\right) \\
\leq & \lambda\left(x_{2 n}, x_{2 n+1}\right)+\frac{\mu d\left(x_{2 n}, T_{1} x_{2 n}\right) d\left(x_{2 n+1}, T_{2} x_{2 n+1}\right)}{d\left(x_{2 n}, T_{2} x_{2 n+1}\right)+d\left(x_{2 n+1}, T_{1} x_{2 n}\right)+d\left(x_{2 n}, x_{2 n+1}\right)} \\
& =\lambda\left(x_{2 n}, x_{2 n+1}\right)+\frac{\mu d\left(x_{2 n}, x_{2 n+1}\right) d\left(x_{2 n+1}, x_{2 n+2}\right)}{d\left(x_{2 n}, x_{2 n+2}\right)+d\left(x_{2 n+1}, x_{2 n+1}\right)+d\left(x_{2 n}, x_{2 n+1}\right)}
\end{aligned}
$$


which implies that

$$
\begin{aligned}
& \left|d\left(x_{2 n+1}, x_{2 n+2}\right)\right| \leq \lambda\left|d\left(x_{2 n}, x_{2 n+1}\right)\right|+\frac{\mu\left|d\left(x_{2 n+1}, x_{2 n+2}\right)\right|}{\left|d\left(x_{2 n}, x_{2 n+2}\right)\right|+\left|d\left(x_{2 n}, x_{2 n+1}\right)\right|}\left|d\left(x_{2 n}, x_{2 n+1}\right)\right| \text {, } \\
& \text { since } \\
& \left|d\left(x_{2 n+1}, x_{2 n+2}\right)\right| \leq\left|d\left(x_{2 n+1}, x_{2 n}\right)\right|+\left|d\left(x_{2 n}, x_{2 n+2}\right)\right| . \\
& \text { Therefore } \\
& \left|d\left(x_{2 n+1}, x_{2 n+2}\right)\right| \leq \lambda\left|d\left(x_{2 n}, x_{2 n+1}\right)\right|+\mu\left|d\left(x_{2 n}, x_{2 n+1}\right)\right| \\
& =(\lambda+\mu)\left|d\left(x_{2 n}, x_{2 n+1}\right)\right|
\end{aligned}
$$

Similarly, we obtain

$\left|d\left(x_{2 n+2}, x_{2 n+3}\right)\right| \leq(\lambda+\mu)\left|d\left(x_{2 n+1}, x_{2 n+2}\right)\right|$

Since $s \lambda+\mu<1$ and $s \geq 1$, we get $\lambda+\mu<1$.

Therefore, with $\delta=\lambda+\mu<1$ and for all $n \geq 0$ and consequently, we have

$$
\begin{aligned}
\left|d\left(x_{2 n+1}, x_{2 n+2}\right)\right| \leq \delta\left|d\left(x_{2 n}, x_{2 n+1}\right)\right| \leq \delta^{2}\left|d\left(x_{2 n-1}, x_{2 n}\right)\right| & \leq \cdots \leq \delta^{2 n+1}\left|d\left(x_{0}, x_{1}\right)\right| \\
\left|d\left(x_{2 n+1}, x_{2 n+2}\right)\right| \leq \delta\left|d\left(x_{2 n}, x_{2 n+1}\right)\right| \leq \delta^{2}\left|d\left(x_{2 n-1}, x_{2 n}\right)\right| & \leq \cdots \leq \delta^{2 n+1}\left|d\left(x_{0}, x_{1}\right)\right|
\end{aligned}
$$

Thus, for any $m>n, m, n \in \mathbb{N}$, we have

$$
\begin{aligned}
& \left|d\left(x_{2 n}, x_{2 m}\right)\right| \leq s\left|d\left(x_{2 n}, x_{2 n+1}\right)\right|+s\left|d\left(x_{2 n+1}, x_{2 m}\right)\right| \\
& \leq s\left|d\left(x_{2 n}, x_{2 n+1}\right)\right|+s^{2}\left|d\left(x_{2 n+1}, x_{2 n+2}\right)\right|+s^{2}\left|d\left(x_{2 n+2}, x_{2 m}\right)\right| \\
& \leq s\left|d\left(x_{2 n}, x_{2 n+1}\right)\right|+s^{2}\left|d\left(x_{2 n+1}, x_{2 n+2}\right)\right|+s^{3}\left|d\left(x_{2 n+2}, x_{2 n+3}\right)\right|+s^{3}\left|d\left(x_{2 n+3}, x_{2 m}\right)\right| \\
& \left|d\left(x_{2 n}, x_{2 m}\right) \dot{\mid} \leq s\right| d\left(x_{2 n}, x_{2 n+1}\right)\left|+s^{2}\right| d\left(x_{2 n+1}, x_{2 n+2}\right)\left|+s^{3}\right| d\left(x_{2 n+2}, x_{2 n+3}\right) \mid+\cdots \\
& \ldots+s^{2 m-2 n-1}\left|d\left(x_{2 m-2}, x_{2 m-1}\right)\right|+s^{2 m-2 n}\left|d\left(x_{2 m-1}, x_{2 m}\right)\right| \\
& \left|d\left(x_{2 n}, x_{2 m}\right)\right| \leq s \delta^{2 n}\left|d\left(x_{0}, x_{1}\right)\right|+s^{2} \delta^{2 n+1}\left|d\left(x_{0}, x_{1}\right)\right|+s^{3} \delta^{2 n+2}\left|d\left(x_{0}, x_{1}\right)\right|+\cdots \\
& \ldots .+s^{2 m-2 n-1} \delta^{2 m-2}\left|d\left(x_{0}, x_{1}\right)\right|+s^{2 m-2 n} \delta^{2 m-1}\left|d\left(x_{0}, x_{1}\right)\right| \\
& =\sum_{i=1}^{2 m-2 n} s^{i} \delta^{i+2 n-1}\left|d\left(x_{0}, x_{1}\right)\right| \text {. }
\end{aligned}
$$

Therefore

$$
\begin{aligned}
\left|d\left(x_{2 n}, x_{2 m}\right)\right| & =\sum_{i=1}^{2 m-2 n} s^{i+2 n-1} \delta^{i+2 n-1}\left|d\left(x_{0}, x_{1}\right)\right| \\
& =\sum_{t=2 n}^{2 m-1} s^{t} \delta^{t}\left|d\left(x_{0}, x_{1}\right)\right| \\
& \leq \sum_{t=2 n}^{\infty}(s \delta)^{t}\left|d\left(x_{0}, x_{1}\right)\right| \\
& =\frac{(s \delta)^{2 n}}{1-(s \delta)}\left|d\left(x_{0}, x_{1}\right)\right|
\end{aligned}
$$

and hence

$$
\left|d\left(x_{2 n}, x_{2 m}\right)\right| \leq \frac{(s \delta)^{2 n}}{1-(s \delta)}\left|d\left(x_{0}, x_{1}\right)\right| \rightarrow 0 \text { as } n, m \rightarrow \infty
$$

Thus, $\left\{x_{n}\right\}$ is a Cauchy sequence in $X$. Since $X$ is complete, there exists some $u \in X$ such that $x_{2 n} \rightarrow u$ as $n \rightarrow \infty$. Suppose that is not possible; then there exists $z \in X$ such that

$$
\left|d\left(u, T_{1} u\right)\right|=|z|>0
$$

So by using the triangular inequality and (21), we get

$$
\begin{aligned}
z & =d\left(u, T_{1} u\right) \\
& \leq s d\left(u, x_{2 n+2}\right)+s d\left(x_{2 n+2}, T u\right) \\
& =s d\left(u, x_{2 n+2}\right)+s d\left(T_{2} x_{2 n+1}, T_{1} u\right) \\
& \leq s d\left(u, x_{2 n+2}\right)+s \lambda d\left(T_{2} x_{2 n+1}, u\right)+\frac{s \mu d\left(x_{2 n+1}, T_{1} x_{2 n+1}\right) d\left(u, T_{2} u\right)}{d\left(x_{2 n+1}, T_{2} u\right)+d\left(u, T_{1} x_{2 n+1}\right)+d\left(x_{2 n+1}, u\right)} \\
& =s d\left(u, x_{2 n+2}\right)+s \lambda d\left(x_{2 n+1}, u\right)+\frac{s \mu d\left(x_{2 n+1}, x_{2 n+2}\right) d\left(u, T_{2} u\right)}{d\left(x_{2 n+1}, T_{2} u\right)+d\left(u, x_{2 n+2}\right)+d\left(x_{2 n+1}, u\right)}
\end{aligned}
$$

which implies that

$|z|=\left|d\left(u, T_{1} u\right)\right|$

$$
\leq s\left|d\left(u, x_{2 n+2}\right)\right|+s \lambda\left|d\left(x_{2 n+1}, u\right)\right|+\frac{s \mu\left|d\left(x_{2 n+1}, x_{2 n+2}\right)\right|\left|d\left(u, T_{1} u\right)\right|}{\left|d\left(x_{2 n+1}, T_{1} u\right)\right|+\left|d\left(u, x_{2 n+2}\right)\right|+\left|d\left(x_{2 n+1}, u\right)\right|}
$$

Taking the limit of (36) as $n \rightarrow \infty$, we obtain that $|z|=\left|d\left(u, T_{1} u\right)\right| \leq 0$, a contradiction with (34).

So $|z|=0$. Hence $T_{1} u=u$.

Similarly $T_{2} u=u$.

Now show that $T_{1}$ and $T_{2}$ have unique common fixed point of $T_{1}$ and $T_{2}$. To show that $u^{*}$ is another fixed point of $T_{1}$ and $T_{2}$.Then,

$$
\begin{aligned}
d\left(u, u^{*}\right) & =d\left(T_{1} u, T_{2} u^{*}\right) \\
& \leq \lambda d\left(u, u^{*}\right)+\frac{\mu d\left(u, T_{1} u\right) d\left(u^{*}, T_{2} u^{*}\right)}{d\left(u, T_{2} u^{*}\right)+d\left(u^{*}, T_{1} u\right)+d\left(u, u^{*}\right)}
\end{aligned}
$$

so that

$$
\left|d\left(u, u^{*}\right)\right| \leq \lambda\left|\quad d\left(u, u^{*}\right)\right|+\frac{\mu\left|\mathrm{d}\left(\mathrm{u}, T_{1} \mathrm{u}\right)\right|\left|\mathrm{d}\left(\mathrm{u}^{*}, T_{2} \mathrm{u}^{*}\right)\right|}{\left|\mathrm{d}\left(\mathrm{u}, T_{2} \mathrm{u}^{*}\right)\right|+\left|\mathrm{d}\left(\mathrm{u}^{*}, T_{1} \mathrm{u}\right)\right|+\left|\mathrm{d}\left(\mathrm{u}, \mathbf{u}^{*}\right)\right|}
$$


$<\lambda\left|d\left(u, u^{*}\right)\right|$, a contradiction.

So $u=u^{*}$, which proves the uniqueness of fixed point $\quad$ in $X$. This complete the proof

Now, we consider the following case: $d\left(x_{2 n}, T_{2} x_{2 n+1}\right)+d\left(x_{2 n+1}, T_{1} x_{2 n}\right)+d\left(x_{2 n}, x_{2 n+1}\right)=0$ (for any $n$ ) implies $d\left(T_{1} x_{2 n}, T_{2} x_{2 n+1}\right)=0$ so that $x_{2 n}=T_{1} x_{2 n}=x_{2 n+1}=T_{2} x_{2 n+1}=x_{2 n+2}$. Thus we have $x_{2 n+1}=$ $T_{1} x_{2 n}=x_{2 n}$, so there exists $K_{1}$ and $l_{1}$ such that $K_{1}=T_{1} l_{1}=l_{1}$. Using foregoing arguments, one can also show that there exists $K_{2}$ and $l_{2}$ such that $K_{2}=T_{2} l_{2}=l_{2}$. As $d\left(l_{1}, T_{2} l_{2}\right)+d\left(l_{2}, T_{1} l_{1}\right)+d\left(l_{1}, l_{2}\right)=0$ (due to definition) implies $d\left(T_{1} l_{1}, T_{2} l_{2}\right)=0, K_{1}=T_{1} l_{1}=T_{2} l_{2}=K_{2}$, which in turn yields that $K_{1}=T_{1} l_{1}=T_{1} K_{1}$. Similarly, one can also have $K_{2}=T_{2} K_{2}$. As $K_{1}=K_{2}$ implies $T_{1} K_{1}=K_{1}$, therefore $K_{1}=K_{2}$ is fixed point of $T$.

We now prove that $T_{1}$ and $T_{2}$ have unique common fixed point. For thus, assume that $K_{1}^{*}$ in $X$ is another fixed point of $T$. Then we have $T_{2} K_{1}^{*}=K_{1}^{*}$. As $d\left(K_{1}, T_{2} K_{1}^{*}\right)+d\left(K_{1}^{*}, T_{1} K_{1}\right)+d\left(K_{1}, K_{1}^{*}\right)=0$, therefore $d\left(K_{1}, K_{1}^{*}\right)=$ $d\left(T_{1} K_{1}, T_{2} K_{1}^{*}\right)=0$.

This implies that $K_{1}=K_{1}^{*}$ which proves the uniqueness of common fixed point in $X$. This completes the proof of the theorem.

\section{References}

[1] R. Kannan, some results on Fixed Points, Bull. Calcutta math. Soc; 60,(1968),71-76.

[2] A. Azam ,B.Fisher and m.khan, common fixed point theorem in complex valued metric space, Numerical complex Analysis \& Optimization 32(3)(2011),243-253.

[3] B.K. Dass and S. Gupta, An extension of Banach contraction principle through rational expressions, Indian J. Pure Appl. Math. 6(1975), 1455-1458

[4] S. Banach, Sur les operation dans les ensembles abstraits et leur application aux equations integrals, Fundamental mathematics 3(1922), 133-181.

[5] S. Bhatt, S. Chaukiyal and R. Dimri, Common fixed point of mappings satisfying rational inequality in complex valued metric spaces, International Journal of Pure and Applied Mathematics 73 (2) (2011),159-164

[6] S. Czerwik, Nonlinear set-valued contraction mappings in $b$-metric spaces, Atti Sem. Mat. Univ.Modena.46(1998), 263-276.

[7] D.S. Jaggi, Some unique fixed point theorems, Indian J.Pure Appl. Math. 8 (1977), 223-230.

[8] S. Datta and S. Ali, A common fixed point theorem under contractive condition in complex valued metric spaces, International Journal of Advanced Scientific and Technical Research 6(2)(2012),467-475.

[9] B.Fisher and M.S. Khan, Fixed points, common fixed points and constant mappings, Studia Sci. Math. Hungar. 11(1978),467-470.

[10] R.K.Verma, Common fixed points in complex valued b-metric spaces satisfying a set of rational equalities ,International journal of mathematics archive -7(10),2016,143-150.

[11] A.A.Mukheimer, Some common fixed point theorems in complex valued $b$-metric spaces, The Scientific World Journal Vol. 2014(2014), Article ID 587825, 6 Pages, http://dx.doi.org/10.1155/2014/587825.

[12] T-zamfirescu, Fixed Point Theorem in metric space, Arch, math.,23,(1974)292-298.

[13] K.P.R. Rao, P.R. Swamy and J.R. Prasad, A common fixed point theorem in complex valued $b$-metric spaces, Bulletin of Mathematics and Statistics Research 1(1) (2013).

[14] J. Roshan, V. Parvanch, S. Sedghi, N. Shobkolaci and W. Shatanawi, Common fixed points of almost generalized (, ’)s-contractive mappings in ordered $b$-metric spaces, Fixed point Theory and Application Vol. 2013(2013):159, pages 1-23, doi:10.1186/16871812-2013-159.

[15] F. Rouzkard and M. Imdad, Some common fixed point theorems on complex valued metric spaces, Computers and Mathematics with Applications 64 (6) (2012), 1866-1874.

[16] .B.E. Rhodes, A comparison of various definitions of contractive mapping, Trans. Amer.Math.Soc.,226, (1977), 257-290.

[17] ] .W. Sintunavarat, Y.J. Cho and P. Kumam, Urysohn integral equations approach by common fixed point in complex valued metric spaces, Advances in Difference Equation Vol. 2013 (December 2013 ):49, pages 1-14.

[18] .W. Sintunavarat and P. Kumam, Generalized common fixed point theorems in complex valued metric spaces and applications, Journal of Inequalities and Application Vol. 2012 (2012) , 84, doi:10.1186/1029-242X-2012-84.

[19] Tanmoy Mitra, A Common fixed point theorem in complex valued b-metric spaces, International Journal of advanced scientific and Technical Research ,available online on http://www.rspublication.com/ijst/index.html.

[20] V. Berinde, On the approximation of fixed points of Contractive Mappings; Carpathion J. Math., 19,(1),(2003), 7-22.

[21] .V. Berinde, On The Conversence of the Ishikawa Iteration in the class of quasi contractive operators, Acta math. Univ. Comenional, 73(1), 2004,119-126.

[22] .V. Berinde, Iterative approximation of fixed points, Lect. Notes maths; val 1992, (2 ${ }^{\text {nd }}$ ed.), Springer verkg, Berlin, 2007.

[23] .A. Beiranvand, S. Moradi, M.Omid and H.Pazandeh, Two fixed point theorem for special mapping, arxiv. 0903.1504VI (Math.FA), 2009.

[24] S. Moradi, Kanan, Fixed Theorem on Complete metric space.al on generalized metric spaces depend on another function arXiv:0903.1577VI [Math.FA].(2009).

[25] .S. K. Chatterjea, Fixed Point Theorem C.R. Acad. Bulgare Sci.,25,(1972),727-730.

[26] I.A. Bakhtin, The contraction principle in quasimetric spaces, Funct. Anal. 30(1989), $26-37$.

[27] .Czerwik, S. Non Linear set valued contractive mappings in b-metric space. Atti. Semin. Mat. Fis. Univ. Modena, 46(1998)263-276.

[28] Czerwik ,S. Krzyszt Dlutekew Singh,S.L., S.L., Round-of stability of iteration procedures for set valued operators in b-metric space; J.Natur.Phy.Sci.,(2001).1-2.

[29] [29] .Czerwik ,S. Krzyszt Dlutekew Singh, S.L., Round off stability of iteration procedures for set valued operators in b-metric space; J.Natur.Phy.Sci.,(2001).1-2.

[30] Aydi, H., Bota, M.F., Karpinar ,E.,and Mitrovic, S. (2012), A fixed point theorems for set valued quasi contration in b-metric space, Fixed Point Theory \& Application.

[31] Boriceanu, M.,(2009), Fixed point theory for multivalued generalized contraction on a set with two b-metric., Babes-Bulyai Uni. Mathematica StudiaLIV.,No.3.1-4. 
[32] .Boriceanu, M., (2009), Fixed Point theory on space with vector valued b-metrics, Demostratio Mathematica Studia, XLII.No.4, 285-301.

[33] Boriceanu, M., (2009), Strict fixed point theorems for multivalued operators in b-metric spaces, Int. I. of modern mathematics 4(2), 285-301

[34] Bota, M. Molnar,A; and Varg, C.,(2011),on eke Lands Variational principle in b-metric spaces, Fixed Point Theory ,12(2),21-28.

[35] Chugh, R.,Kumar, V. and Kadian ,T.,(2012), Some fixed point theorem for multivalued mappings in generalized b-metric spaces ,Int.J. of Mathematical Archive 3(3),1198-1210.

[36] Kir, M. and Kiziltune, H.,(2013),on some well known fixed point theorem in b-metric spaces, Turkish Journal of Analysis and number theory $1(1), 13-16$.

[37] Olaru, I.M. and Branga, A., (2011), Common fixed results in b-k-metric space .General mathematics, 19(4), 51-59.

[38] Olatinwo, M. O. and Imoru, Christopher, O,(2008), A generalization of some results on multivalued weakly picard mappings in bmetric space, Fascienli-mathematici,40,45-46.

[39] Pacurar, m.,(2010), A fixed point result for $\psi$-contrational on b-metric spaces without the boundness assumption, Fascienlimathematici,43,125-137.

[40] Pacurar, M.,(2010), Sequence of almost contractions and fixed points in b-metric spaces, Analek Universitatc de vest, Timi Soara, Seria mathematica Informatica XLVIII,3,125-137.

[41] Rao, K.P.R, and Rao, K.R.K.,(2013), A Common fixed point theorem for two hybrid points of mappings in b-metric spaces, Int.J.Anal,40-38.

[42] Roshan, T.R., Parvanch, V., Sdghi,S., Shubkolaci, N. and Shatanawi,W.,(2013), Common fixed points of almost generalized ( $\psi$, $\psi$ ) s-Contractive mappings in ordered b-metric spaces. Fixed point theorem and application , 159.

[43] Akkouchi, M. (2011), A common fixed point theorem for expansive mappings under strict implicitecondition on b-metric spaces, Acta, Uni. Pdecki, olomac Fac. Rer mathematica50,5-15.

[44] Pankaj ,etat (2014), some fixed point theorems in b-metric space, Turkish J. of Analysis nd number theory, 2(1),19-22.

[45] Dubey, A.K., Shukla, R. and Dubey, R.P. (2014), Some fixed point results in b-metric space, Asian journal of mathematics and application, Vol 2014, Article ID. Amao147, 6pages.

[46] Agrawal, S.,Qureshi, K. and Jyoti, nema (2016), A fixed point theorem for b-metric space, Int. J. of Pure and Applied mathematical Sciences, Vol 9(1), 45-50.

[47] Tiwari, S.K. and S.Dewangan, (2017) B-metic space and common fixed point theorem for contractive mappings, Int. J. of mathematical Archive, 8(3), 1-6.

[48] Dubey, A.K.,R. Shukla and Dubey, R.P. (2015), Some fixed point theorem in complex valued b-metric spaces, Journal of complex system, Vol 2015, Article ID 832467, 7 pages. 